\title{
Tolerance and safety of intravenous streptomycin therapy in patients with tuberculosis
}

R. Pérez Tanoira; F. Sánchez-Patán; A. Jiménez Girón; R. Fernández Roblas; J. Esteban; M. L. Fernández Guerrero

Although the clinical use of streptomycin has declined greatly, it is still a commonly used anti-tuberculous drug. Given the re-emergence of tuberculosis and the limited number of anti-tuberculous drugs, the use of streptomycin has gained renewed interest.

Streptomycin is administered by deep intramuscular injections and, in both Europe and the USA, the manufacturers do not recommend intravenous administration. However, repeated intramuscular injections of streptomycin produce pain and inflammation.

In this investigation, we assessed the tolerance and safety of intravenous streptomycin, an off-label method of administration that could be clinically useful in patients with tuberculosis and other infectious diseases.

Consecutive patients with tuberculosis who were B50 years old and had normal renal function (serum creatinine $11.2 \mathrm{mg} / \mathrm{dl}$ ) were selected. The study was conducted in a university-affiliated hospital from January 2011 to March 2012. This study was approved by the local institutional committee of clinical trials. The purpose of the study was explained to the patients and those that gave written informed consent were included.

Patients were empirically treated with standard doses of rifampin, isoniazid and pyrazinamide. In addition, streptomycin sulphate (Estreptomicina Normon, Madrid, Spain) $15 \mathrm{mg} / \mathrm{kg} / 24 \mathrm{~h}$ in $100 \mathrm{ml}$ of normal saline was administered

intravenously over 45-60 min. In some patients with risk factors for multi-resistant tuberculosis, ethambutol was also added in the initial empirical regimen.

Serum levels of streptomycin were measured on the third day of treatment by means of ultra-performance liquid chromatography-mass spectrometry $1 \mathrm{~h}$ after the end of infusion (peak level) and before the next dose (trough

level), following a protocol previously detailed.

Patients were examined daily with bedside vestibular and Romberg tests and asked for signs of vestibular or cochlear dysfunction. Haematological testing and chemistry were recorded at baseline and every other day. Screen for hearing loss was assessed by a Rinne and Weber $256-\mathrm{Hz}$ tuning fork at the bedside to avoid movements of contagious patients through the hospital. When tests were uncertain or responses difficult to reproduce, pure tone audiometry was performed. Because the study was designed to assess the tolerance of intravenous streptomycin therapy in the short term, we considered a minimum of 1 week of administration in order to include patients into the analysis. Basically, streptomycin was stopped when rapid, DNA-based, molecular tests to detect mutations associated with drug resistance indicated full susceptibility of the infecting strain to rifampin and isoniazid, when sputum acid-fast smears were negative or when the health-care provider estimated that the patient could be discharged from hospital to follow therapy on an outpatient basis. Some of these patients continued intramuscular streptomycin therapy for different periods of time. 
Twenty-seven patients were given intravenous streptomycin. Four of them received another course of streptomycin therapy in subsequent admissions; hence, 31 courses of streptomycin therapy were finally assessed. Most patients $(89 \%)$ were men with pulmonary $(70 \%)$ or disseminated tuberculosis (30\%). The mean age was $36.5 \pm 10.5$ years. Patients had a diagnosis of tuberculosis-based clinical findings, imaging techniques and acidfast smears and/or cultures. Fifty-two percent of patients were HIV-positive individuals and $40 \%$ were immigrants from South America. Intravenous streptomycin was given from 6 to 23 days ( $11.1 \pm 4.5$ days). Mean \pm standard deviation (SD) peak and trough levels were $39.8 \pm 21.6$ and $2.0 \pm$ $0.3 \mathrm{lg} / \mathrm{ml}$, respectively. The 75th percentile was 45.9 and $2.2 \mathrm{lg} / \mathrm{ml}$ at peak and trough, respectively.

Patients tolerated intravenous streptomycin without vestibular or cochlear toxicity. Three patients developed circumoral paresthesia during or immediately after streptomycin infusion that ceased when the rate of infusion was slowed down and administering the dose did not take less than $45 \mathrm{~min}$. Serum creatinine levels did not change after treatment $(0.8 \pm 0.2$ vs. $0.9 \pm 0.3 \mathrm{mg} / \mathrm{dl})$. Changes in hearing capacity were not observed. In five patients with uncertain Rinne and Weber tuning fork tests, the audiograms did not show significant abnormalities. Phlebitis or fever associated with intravenous catheters was not observed.

Soon after the introduction of streptomycin in clinical practice, the general recommendation was made that the antibiotic should be given by intramuscular injection or by slow intravenous infusion. However, the association of intravenous administration, high serum levels of the drug and an increase in side effects led to the decision to recommend intramuscular injection of streptomycin. This belief has remained until now and providers do not recommend intravenous administration of streptomycin.

Cochlear and vestibular dysfunction are the main and more severe side effects observed during streptomycin therapy. In studies performed to compare the incidences of toxicities among patients with tuberculosis receiving different aminoglycoside drugs, streptomycin, regardless of the route of administration, was less ototoxic than kanamycin or amikacin. In these studies, ototoxicity was not associated with the route of administration, but it was associated with older age, longer duration of treatment and greater total dose received. Noteworthy, streptomycin, considered to be the least nephrotoxic aminoglycoside, determined most cases of renal dysfunction, although it was mild and short-lived. To the best of our knowledge, only a few cases of tuberculosis and endocarditis caused by high-level gentamicin-resistant Enterococcus faecalis have been treated with intravenous streptomycin. Peloquin et al. treated a series of 32 patients with tuberculosis with intravenous streptomycin and showed that the drug was

well tolerated when administered daily or thrice-weekly and no more toxic that other aminoglycoside drugs.

In this study, we found that the drug was well tolerated and only paresthesia in and around the mouth was noted in a few patients. Serum levels of streptomycin were in the range previously reported in patients treated with streptomycin $15 \mathrm{mg} / \mathrm{kg}$ per day and, noteworthy, trough levels of streptomycin were below the previously established toxic threshold. 
In the hospital setting, intravenous administration of streptomycin may be helpful and a more convenient route for administration, preventing the complications and discomfort of repeated intramuscular injections. We believe that intravenous administration of streptomycin is safe and well tolerated, and may definitely be considered for the treatment of tuberculosis and other infections in the hospitalised patient. 